\title{
Comparative Study of the Natural Fiber and Artificial Fibre as an Admixture in M30 Grade Concrete
}

\author{
Ch. Mallika Chowdary, I. Siva Kishore
}

\begin{abstract}
The Sugarcane Bagasse which is produced in tones every year which can cause inconvenience to environment, we use this material as one of the admixtures in the concrete mix to reduce its effect on surroundings. As we add the material, we can economize the total expenditure on the construction. By using this we are able to improve the ordinary Portland cement through means of strength which can rise the durability of the structure. By using these materials in proportions, we produce various nominal conventional concrete mixes which are very economical and suitable for any environmental conditions. We want to use the sugarcane bagasse as the main source is an eco-friendly material, but only with this material we can't get good strength hence it imparts strength to the concrete we are using the combination of the sugar cane bagasse and the optical fiber as an admixture. Here the test results give good improvement. Hence low-cost materials with good strength is obtained.
\end{abstract}

Keywords: Artificial fibres, Bagasse ash, Compressive strength, Natural fibres, plastic optical fibre.

\section{INTRODUCTION}

The utilization of by-products in the production of concrete has gained considerable interest among concrete technologists in the recent years. Sugarcane Bagasse (SCB) is an agro waste obtained from sugar industry. i.e., fibrous waste obtained after the extraction of juice. It exhibits pozzolanic property. Bagasse is used as a biofuel and is also used in researches. That is usually incorporated into concrete mixes to produce concrete with exception properties. Ash is obtained after the burning of the bagasse. Every year millions of tons of ash are generated from thermal power plants. In additional to this a larger quantity of agriculture waste like rice husk ash, sugarcane bagasse ash is also produced. The problem gets compounded with million tons of waste being generated worldwide inform of demolished waste from natural and technological disasters. There is a growing concern to limit the amount of waste by recycling which will provide opportunities for saving energy, time and resources.

Revised Manuscript Received on October 10, 2020.

*Correspondence Author

Ch. Mallika Chowdary*, Assistant Professor, Department of Civil Engineering, Koneru Lakshmaiah Educational Foundation, Vaddeswaram, Guntur, A.P, India. Contac mail: chmallika@kluniversity.in

I. Siva Kishore, Assistant Professor, Department of Civil Engineering, Koneru Lakshmaiah Educational Foundation, Vaddeswaram, Guntur, A.P, India. Contac mail: i.sivakishore@kluniversity.in

(c) The Authors. Published by Blue Eyes Intelligence Engineering and Sciences Publication (BEIESP). This is an open access article under the CC BY-NC-ND license (http://creativecommons.org/licenses/by-nc-nd/4.0/)

\section{A. Plastic optical fiber}

The plastic optical is a flexible light guide rod (flexible lightning transmitting strip). Plastic Optical fiber is extremely durable and is able to be bent much more than glass fiber, however it is flammable. Conventional Plastic Optical fibers are made from glass materials \& widely used as optical signal-transmitting mediums for instrumentation between instruments or in an instrument, for transmission of data, for medical use, for decoration, for transmission of image.

\section{B. Sugarcane Bagasse}

The Bagasse is the dry pulpy residue left after the extraction of juice from sugarcane. Mineral admixture like sugar cane bagasse ash is most common type of by-product. The SCB consists of $50 \%$ of cellulose, $25 \%$ of hemi-cellulose \& $25 \%$ of lignin. Each ton of sugarcane generates approximately $26 \%$ of bagasse (at a moisture content of 50\%) and $0.62 \%$ of residual ash. Bagasse is a heterogeneous material containing around 30-40 \% of "pith", "bast", "rind", "stem" fiber. This property makes bagasse as an admixture with the conventional concrete used in cement, fine aggregate, coarse aggregate and water. The residue after combustion presents a chemical composition dominates by silicon dioxide ( $\mathrm{SiO} 2)$. In spite of being a material of hard degradation and that presents few nutrients. The ash is used in the farms as a fertilizer in the sugarcane harvests. Bagasse is the essential ingredient for the production of pressed building board, acoustical and other construction material and can be made into a number of biodegradable plastics. Bagasse is also employed in the production of furfural, a clear colorless liquid used in the synthesis of chemical products such as nylons, solvents and even medicines. Bagasse is readily available as a waste product with a high sugar content and as a potential as an environmentally friendly alternative to corn as a source of the biofuel ethanol (ethyl alcohol).

\section{METHODOLOGY}

The experimental investigation or methodology consist of casting and testing one series of plain concrete and three series of concrete specimens with varying amounts admixtures is prepared and tested. 


\section{Comparative Study of the Natural Fiber and Artificial Fibre as an Admixture in M30 Grade Concrete}

A. Mix Design: Suggests concrete the mix design processes for both air-entrained and non-air entrained concrete. Both the methods are based on the following principles.

- The workability of the mix depends on the water content and max size of aggregates.

- The water cement ratio (w/c ratio) is solely dependent upon the design strength with a restriction from the durability point of view. The w/c ratio is inversely proportional to the design strength.

- The bulk volume of coarse aggregate per unit volume of concrete depends on the max size of the coarse aggregate and the grading of the fine aggregate, expressed as the fineness modulus.

The design starts with the selection of the water content for a given max size of coarse aggregate and the workability required for the type of work, with workability being expressed by slump. Cement content is the found out simple from this water content and the $\mathrm{w} / \mathrm{c}$ ratio, determine earlier on the basis of the design strength. The volume of coarse aggregate is then determined, and fine aggregate content is found out by subtracting the volume of other ingredients from the total volume of concrete. The basis is a trial and error approach while the volume raises is more direct and gives more accurate result.

\section{B. Procedure}

Preparation of Admixture, Sugarcane bagasse and Plastic optical fibre is made into pieces and into stripes of length $5.5 \mathrm{~mm}$ having a diameter of $0.80 \mathrm{~mm}$.

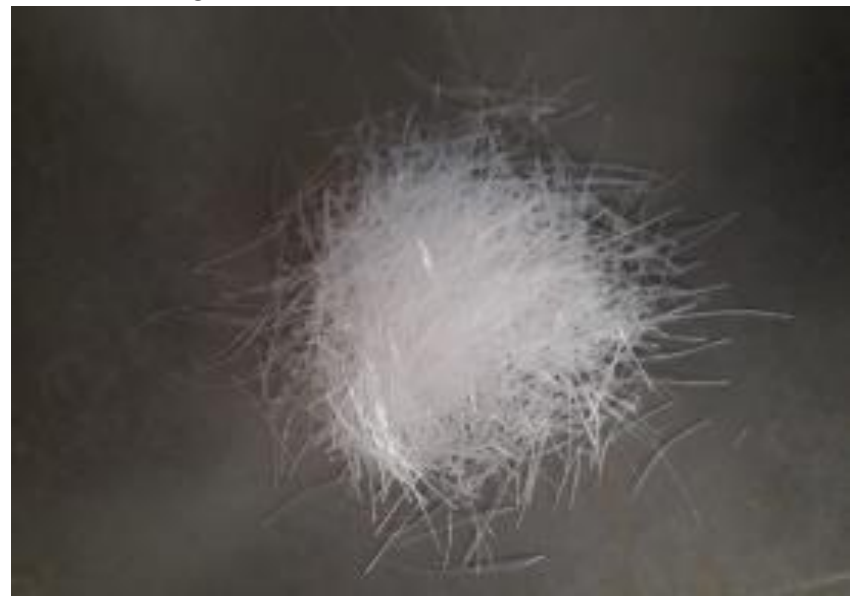

Fig (2.1) Preparation of Optical Fibre

\section{Preparation of Cubes and Cylinders}

Before starting the mixing, the coarse aggregate, fine aggregate, cement and required mixing water was added in the order. Admixture, sugarcane bagasse and plastic optical fiber is added at the same time with cement and fine aggregate. After all of the ingredients has been added, they are thoroughly mixed for 5 minutes followed by a brief rest period to confirm if the mixture was workable, then run for an additional 2 minutes. Then slump test was carried out following IS: 1199-1959.The moulds used were $150 \mathrm{~mm} * 150 \mathrm{~mm} * 150 \mathrm{~mm}$ rigid steel forms. The moulds where filled with three lifts of freshly mixed concrete, tamping each lift 25 times with tamping rod and tapping each lift lightly with mallet 10 to 15 times. The excess concrete was struck off and finished to a smooth surface with steel or wooden trowel. The moulded cubes were left covered at room temperature for about 24 hours after which the moulds.

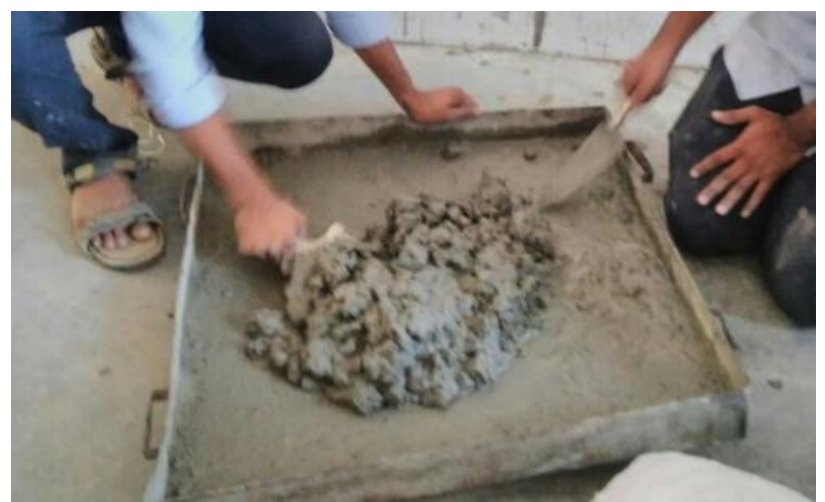

Fig (2.2) Preparation of Concrete Mixing

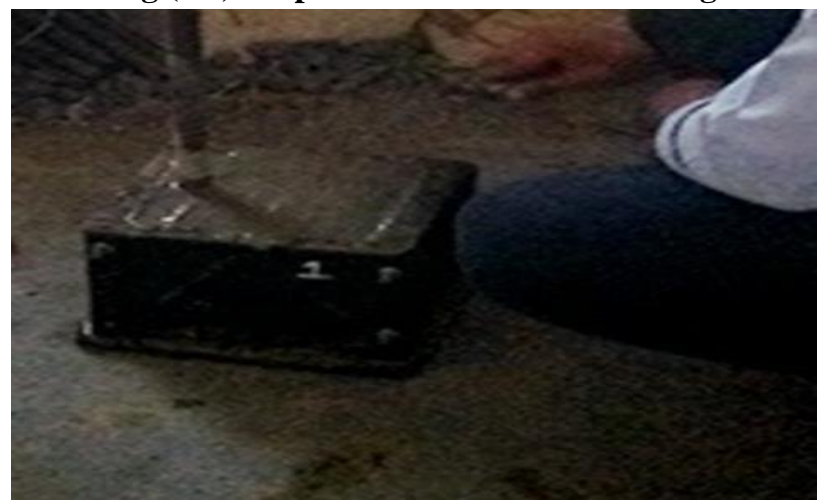

Fig (2.3) Casting of Cube

D. Curing of Concrete: The mould cubes were removed and then the cubes were transferred to curing tank, set at a temperature of 26c and relatively humidity of approximately $95-100 \%$. The cubes were used to test for compressive strength and split tensile strength. One set of three cubes was tested after 7 days and another set of three tested after 14 days and another for 28days.

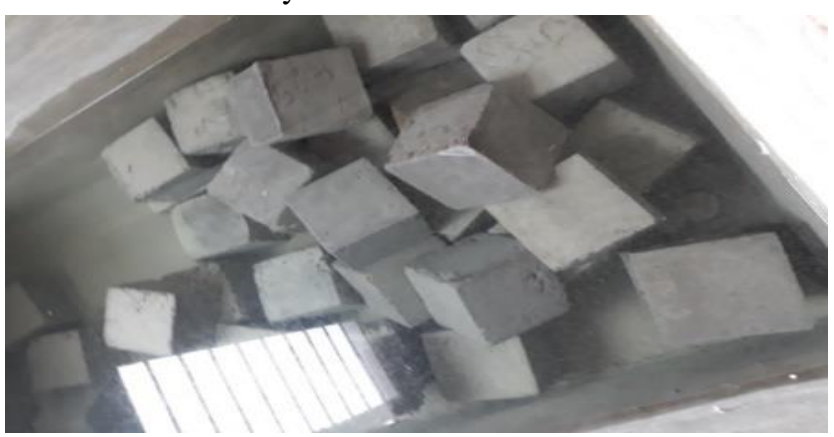

Fig (2.4) Curing of Cubes in Tank

E. Compressive Strength: The specimens (cubes) of size $150 \mathrm{~mm}$ x $150 \mathrm{~mm}$ x $150 \mathrm{~mm}$ that were prepared for each mix were remoulded after 24 hours and cured for 7,14 \& 28 days were tested in compressive testing machine. The compressive strength results obtained from the three identical cubes is taken average of it.

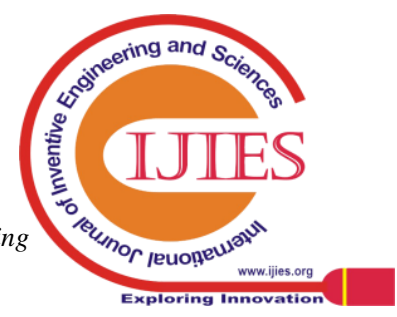




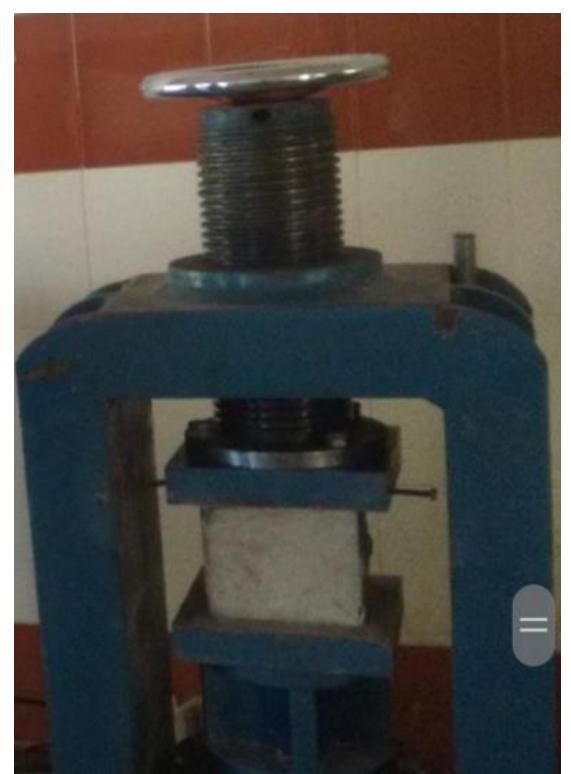

Fig (2.5) Compressive Testing Machine

\subsubsection{Split Tensile Strength}

The Cylinders of specimen of the size $150 \mathrm{~mm}$ x $300 \mathrm{~mm}$ that were prepared for each mix were remoulded and cured for 7,14 \& 28 days.

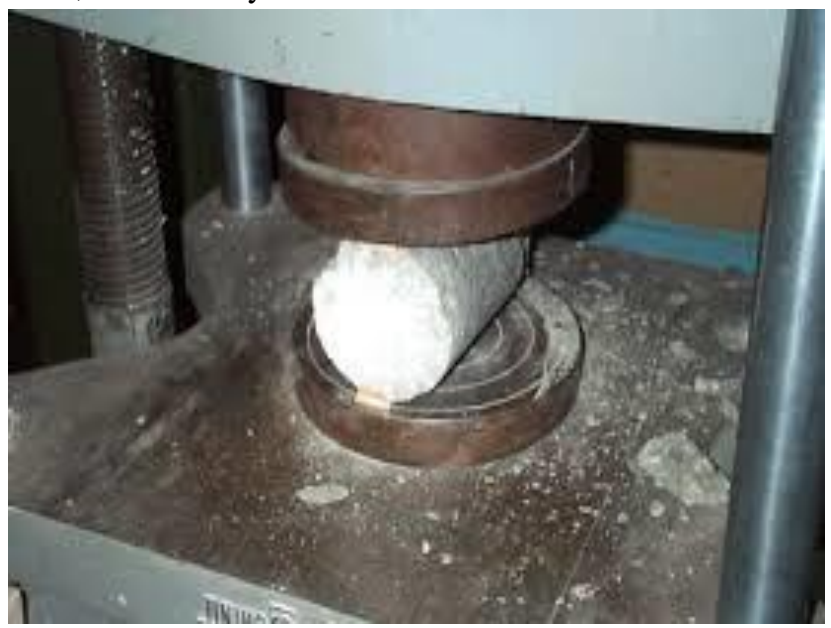

Fig (2.6) Split tensile Strength Test

F. double-blind review process, which means that both the reviewer (s) and author (s) identities concealed from the reviewers, and vice versa, throughout the review process. All submitted manuscripts are reviewed by three reviewer one from India and rest two from overseas. There should be proper comments of the reviewers for the purpose of acceptance/ rejection. There should be minimum 01 to 02 week time window for it.

\section{MATH}

If you are using Word, use either the Microsoft Equation Editor or the MathType add-on (http://www.mathtype.com) for equations in your paper (Insert | Object | Create New | Microsoft Equation or MathType Equation).

"Float over text" should not be selected.

\section{PREPARE YOUR PAPER BEFORE STYLING}

Before you begin to format your paper, first write and save the content as a separate text file. Complete all content and organizational editing before formatting. Please note sections A-D below for more information on proofreading, spelling and grammar. Keep your text and graphic files separate until after the text has been formatted and styled. Do not use hard tabs, and limit use of hard returns to only one return at the end of a paragraph. Do not add any kind of pagination anywhere in the paper. Do not number text heads-the template will do that for you.

\section{A. Abbreviations and Acronyms}

Define abbreviations and acronyms the first time they are used in the text, even after they have been defined in the abstract. Abbreviations such as IEEE, SI, MKS, CGS, sc, dc, and rms do not have to be defined. Do not use abbreviations in the title or heads unless they are unavoidable.

\section{B. Units}

- Use either SI (MKS) or CGS as primary units. (SI units are encouraged.) English units may be used as secondary units (in parentheses). An exception would be the use of English units as identifiers in trade, such as "3.5-inch disk drive".

- Avoid combining SI and CGS units, such as current in amperes and magnetic field in oersteds. This often leads to confusion because equations do not balance dimensionally. If you must use mixed units, clearly state the units for each quantity that you use in an equation.

- Do not mix complete spellings and abbreviations of units: "Wb/m2" or "webers per square meter", not "webers/m2". Spell out units when they appear in text: ". . . a few henries", not ". . . a few H".

\section{Equations}

The equations are an exception to the prescribed pacifications of this template. You will need to determine whether or not your equation should be typed using either the Times New Roman or the Symbol font (please no other font). To create multileveled equations, it may be necessary to treat the equation as a graphic and insert it into the text after your paper is styled.

Number equations consecutively. Equation numbers, within parentheses, are to position flush right, as in (1), using a right tab stop. To make your equations more compact, you may use the solidus ( / ), the exp function, or appropriate exponents. Italicize Roman symbols for quantities and variables, but not Greek symbols. Use a long dash rather than a hyphen for a minus sign. Punctuate equations with commas or periods when they are part of a sentence, as in:

$$
a+b=\gamma
$$

Note that the equation is centered using a center tab stop. Be sure that the symbols in your equation have been defined before or immediately following the equation. Use "(1)", not "Eq. (1)" or "equation (1)", except at the beginning of a sentence: "Equation (1) is ..."

\section{Figures and Tables}

- Positioning Figures and Tables: Place figures and tables at the top and bottom of columns. Avoid placing them in the middle of columns. Large figures and tables may span across both columns.

Published By:

Blue Eyes Intelligence Engineering \& Sciences Publication (C) Copyright: All rights reserved.

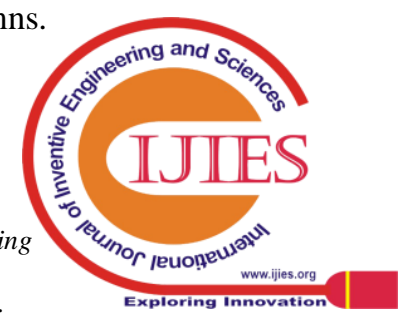


Figure captions should be below the figures; table heads should appear above the tables. Insert figures and tables after they are cited in the text. Use the abbreviation "Fig. 1", even at the beginning of a sentence.

Table- II: Name of the Table that justify the values

\begin{tabular}{|l|l|c|c|}
\hline \multirow{2}{*}{$\begin{array}{c}\text { Table } \\
\text { Head }\end{array}$} & \multicolumn{3}{|c|}{ Table Column Head } \\
\cline { 2 - 4 } copy & Table column subhead & Subhead & Subhead \\
\hline \multicolumn{3}{|c|}{ a. Sable copy ${ }^{\mathrm{a}}$} & \\
\hline
\end{tabular}

The figure, graph, chart can be written as per given below schedule.

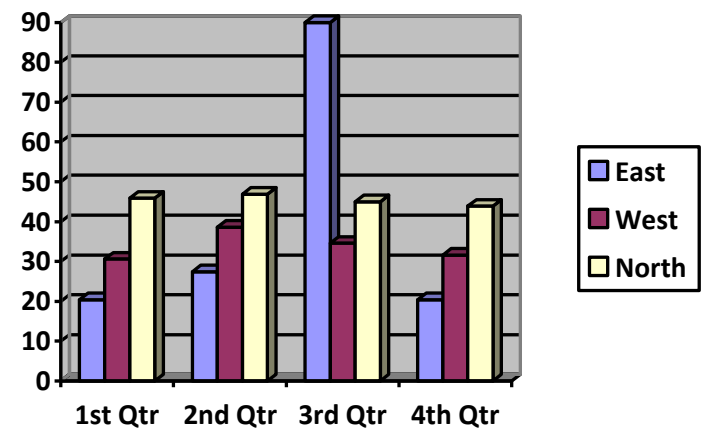

Fig. 1.Example of a figure caption. (figure caption)

\section{HELPFUL HINTS}

\section{A. Figures and Tables}

Because the final formatting of your paper is limited in scale, you need to position figures and tables at the top and bottom of each column. Large figures and tables may span both columns. Place figure captions below the figures; place table titles above the tables. If your figure has two parts, include the labels "(a)" and "(b)" as part of the artwork. Please verify that the figures and tables you mention in the text actually exist. Do not put borders around the outside of your figures. Use the abbreviation "Fig." even at the beginning of a sentence. Do not abbreviate "Table." Tables are numbered with Roman numerals. Include a note with your final paper indicating that you request color printing.

You can use color figures as per the requirement but fonts should be in black. Authors can use any number of color diagram, chart, picture, screenshots, and any snap which is required for the research of the title.

Table- II: Name of the Table that justify the values

\begin{tabular}{|c|c|c|c|c|c|c|c|c|}
\hline $\mathrm{A}$ & $\mathrm{B}$ & $\mathrm{C}$ & $\mathrm{D}$ & $\mathrm{E}$ & $\mathrm{G}$ & $\mathrm{I}$ & $\mathrm{J}$ & $\mathrm{K}$ \\
\hline Value & & & & & & & & \\
\hline Value & & & & & & & & \\
\hline Value & & & & & & & & \\
\hline & & & & & & & & \\
\hline
\end{tabular}

Figure axis labels are often a source of confusion. Use words rather than symbols. As an example, write the quantity "Magnetization," or "Magnetization $M$," not just " $M$." Put units in parentheses. Do not label axes only with units. As in Fig. 1, for example, write "Magnetization $(\mathrm{A} / \mathrm{m})$ " or "Magnetization $\left(\mathrm{A} \cdot \mathrm{m}^{-1}\right)$," not just “A/m." Do not label axes with a ratio of quantities and units. For example, write "Temperature (K)," not "Temperature/K."

Multipliers can be especially confusing. Write "Magnetization $(\mathrm{kA} / \mathrm{m})$ ") or "Magnetization $\left(10^{3} \mathrm{~A} / \mathrm{m}\right)$." Do not write "Magnetization $(\mathrm{A} / \mathrm{m}) \times 1000$ " because the reader would not know whether the top axis label in Fig. 1 meant $16000 \mathrm{~A} / \mathrm{m}$ or $0.016 \mathrm{~A} / \mathrm{m}$. Figure labels should be legible, approximately 8 to 12 point type.

\section{B. References}

Number citations consecutively in square brackets [1]. The sentence punctuation follows the brackets [2]. Multiple references [2], [3] are each numbered with separate brackets [1]-[3]. When citing a section in a book, please give the relevant page numbers [2]. In sentences, refer simply to the reference number, as in [3]. Do not use "Ref. [3]" or "reference [3]" except at the beginning of a sentence: "Reference [3] shows ... ." Number footnotes separately in superscripts (Insert | Footnote). ${ }^{1}$ Place the actual footnote at the bottom of the column in which it is cited; do not put footnotes in the reference list (endnotes). Use letters for table footnotes (see Table I).

Please note that the references at the end of this document are in the preferred referencing style. Give all authors' names; do not use "et al." unless there are six authors or more. Use a space after authors' initials. Papers that have not been published should be cited as "unpublished" [4]. Papers that have been submitted for publication should be cited as "submitted for publication" [5]. Papers that have been accepted for publication, but not yet specified for an issue should be cited as "to be published" [6]. Please give affiliations and addresses for private communications [7].

Capitalize only the first word in a paper title, except for proper nouns and element symbols. For papers published in translation journals, please give the English citation first, followed by the original foreign-language citation [8].

\section{Abbreviations and Acronyms}

Define abbreviations and acronyms the first time they are used in the text, even after they have already been defined in the abstract.

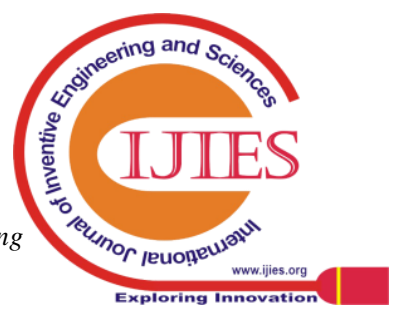


Abbreviations such as SI, ac, and dc do not have to be defined. Abbreviations that incorporate periods should not have spaces: write "C.N.R.S.," not "C. N. R. S." Do not use abbreviations in the title unless they are unavoidable (for example, "Journal Name" in the title of this article).

\section{Equations}

Number equations consecutively with equation numbers in parentheses flush with the right margin, as in (1). First use the equation editor to create the equation. Then select the "Equation" markup style. Press the tab key and write the equation number in parentheses. To make your equations more compact, you may use the solidus ( / ), the exp function, or appropriate exponents. Use parentheses to avoid ambiguities in denominators. Punctuate equations when they are part of a sentence, as in

$$
\begin{array}{rl}
\int_{0}^{r_{2}} & F(r, \varphi) d r d \varphi=\left[\sigma r_{2} /\left(2 \mu_{0}\right)\right] \\
& \cdot \int_{0}^{\infty} \exp \left(-\lambda\left|z_{j}-z_{i}\right|\right) \lambda^{-1} J_{1}\left(\lambda r_{2}\right) J_{0}\left(\lambda r_{i}\right) d \lambda .
\end{array}
$$

Be sure that the symbols in your equation have been defined before the equation appears or immediately following. Italicize symbols ( $T$ might refer to temperature, but $\mathrm{T}$ is the unit tesla). Refer to "(1)," not "Eq. (1)" or "Equation (1) is ...."

\section{E. Other Recommendations}

Use one space after periods and colons. Hyphenate complex modifiers: "zero-field-cooled magnetization." Avoid calculated." [It is not clear who or what used (1).] Write "Using (1), we calculated the potential."

Use a zero before decimal points: " 0.25 ," not ".25." Use " $\mathrm{cm}^{3}$," not "cc." Indicate sample dimensions as " $0.1 \mathrm{~cm} \times 0.2$ cm," not " $0.1 \times 0.2 \mathrm{~cm}^{2}$." The abbreviation for "seconds" is "s," not "sec." Do not mix complete spellings and abbreviations of units: use " $\mathrm{Wb} / \mathrm{m}^{2}$ " or "webers per square meter," not "webers $/ \mathrm{m}^{2}$." When expressing a range of values, write "7 to 9" or "7-9," not "7 9."

A parenthetical statement at the end of a sentence is punctuated outside of the closing parenthesis (like this). (A parenthetical sentence is punctuated within the parentheses.) In American English, periods and commas are within quotation marks, like "this period." Other punctuation is "outside"! Avoid contractions; for example, write "do not" instead of "don't." The serial comma is preferred: "A, B, and C" instead of "A, B and C."

If you wish, you may write in the first person singular or plural and use the active voice ("I observed that ..." or "We observed that ..." instead of "It was observed that ..."). Remember to check spelling.

If your native language is not English, please get a native English-speaking colleague to proofread your paper.

\section{RESULT AND DISCUSSION}

The contents of the journal are peer-reviewed and archival. The journal publishes scholarly articles of archival value as well as tutorial expositions and critical reviews of classical subjects and topics of current interest. "equation (1)," except at the beginning of a sentence: dangling participles, such as, "Using (1), the potential was instead, "The potential was calculated by using (1)," or

Authors should consider the following points:

1) Technical papers submitted for publication must advance the state of knowledge and must cite relevant prior work.

2) The length of a submitted paper should be commensurate with the importance, or appropriate to the complexity, of the work. For example, an obvious extension of previously published work might not be appropriate for publication or might be adequately treated in just a few pages.

3) Authors must convince both peer reviewers and the editors of the scientific and technical merit of a paper; the standards of proof are higher when extraordinary or unexpected results are reported.

4) Because replication is required for scientific progress, papers submitted for publication must provide sufficient information to allow readers to perform similar experiments or calculations and use the reported results. Although not everything need be disclosed, a paper must contain new, useable, and fully described information. For example, a specimen's chemical composition need not be reported if the main purpose of a paper is to introduce a new measurement technique. Authors should expect to be challenged by reviewers if the results are not supported by adequate data and critical details.

\section{CONCLUSION}

A conclusion section is not required. Although a conclusion may review the main points of the paper, do not replicate the abstract as the conclusion. A conclusion might elaborate on the importance of the work or suggest applications and extensions.

\section{APPENDIX}

It is optional. Appendixes, if needed, appear before the acknowledgment.

\section{ACKNOWLEDGMENT}

It is optional. The preferred spelling of the word "acknowledgment" in American English is without an "e" after the "g." Use the singular heading even if you have many acknowledgments. Avoid expressions such as "One of us (S.B.A.) would like to thank ...." Instead, write "F. A. Author thanks" Sponsor and financial support acknowledgments are placed in the unnumbered footnote on the first page.

\section{REFERENCES}

1. G. O. Young, "Synthetic structure of industrial plastics (Book style with paper title and editor)," in Plastics, 2nd ed. vol. 3, J. Peters, Ed. New York: McGraw-Hill, 1964, pp. 15-64.

2. W.-K. Chen, Linear Networks and Systems (Book style). Belmont, CA: Wadsworth, 1993, pp. 123-135.

3. H. Poor, An Introduction to Signal Detection and Estimation. New York: Springer-Verlag, 1985, ch. 4.

4. B. Smith, "An approach to graphs of linear forms (Unpublished work style)," unpublished.

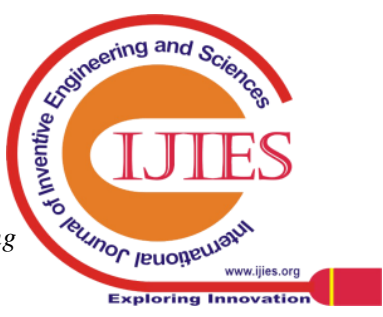


5. E. H. Miller, "A note on reflector arrays (Periodical style-Accepted for publication)," IEEE Trans. Antennas Propagat., to be published.

6. J. Wang, "Fundamentals of erbium-doped fiber amplifiers arrays (Periodical style-Submitted for publication)," IEEE J. Quantum Electron., submitted for publication.

7. C. J. Kaufman, Rocky Mountain Research Lab., Boulder, CO, private communication, May 1995.

8. Y. Yorozu, M. Hirano, K. Oka, and Y. Tagawa, "Electron spectroscopy studies on magneto-optical media and plastic substrate interfaces(Translation Journals style)," IEEE Transl. J. Magn.Jpn., vol. 2, Aug. 1987, pp. 740-741 [Dig. $9^{\text {th }}$ Annu. Conf. Magnetics Japan, 1982, p. 301].

9. M. Young, The Techincal Writers Handbook. Mill Valley, CA: University Science, 1989.

10. (Basic Book/Monograph Online Sources) J. K. Author. (year, month, day). Title (edition) [Type of medium]. Volume(issue). Available: http://www.(URL)

11. J. Jones. (1991, May 10). Networks (2nd ed.) [Online]. Available: http://www.atm.com

12. (Journal Online Sources style) K. Author. (year, month). Title. Journal [Type of medium]. Volume(issue), paging if given. Available: http://www.(URL) 